\title{
Pulmonary Adenocarcinoma Transforming into Small Cell Carcinoma: An Extreme Rarity
}

\author{
Chhabra $\mathrm{S}^{1}$, Bhutani $\mathrm{N}^{\star 2}$, Sangwan $\mathrm{M}^{3}$, Agarwal $\mathrm{M}^{4}$, Chhabra $\mathrm{R}^{5}$ and Sen $\mathrm{R}^{6}$ \\ ${ }^{1}$ Professor, Department of Pathology, PGIMS Rohtak, Haryana, India \\ ${ }^{2}$ Resident, Department of Pathology, PGIMS Rohtak, Haryana, India \\ ${ }^{3}$ Senior Resident, Department of Pathology, PGIMS Rohtak, Haryana, India \\ ${ }^{4}$ Resident, Department of Pathology, PGIMS Rohtak, Haryana, India \\ ${ }^{5}$ Resident, Department of Pathology, PGIMS Rohtak, Haryana, India \\ ${ }^{6}$ Senior Professor and Head, Department of Pathology, PGIMS Rohtak, Haryana, India
}

*Corresponding author: Bhutani N, Resident, Department of Pathology, PGIMS Rohtak, Haryana, India, Fax: +91-1262-211308, Tel: +91-9873101393, E-mail: namitabhutani89@gmail.com

Citation: Chhabra S, Bhutani N, Sangwan M, Agarwal M, Chhabra R, et al. (2016) Pulmonary Adenocarcinoma Transforming into Small Cell Carcinoma: An Extreme Rarity. J Cancer Sci Clin Oncol 3(2): 205. doi: 10.15744/2394-6520.3.205

Received Date: June 30, 2016 Accepted Date: September 09, 2016 Published Date: September 12, 2016

\begin{abstract}
Primary small cell lung cancer (SCLC) showing epidermal growth factor receptor (EGFR) mutation is extremely rare. Transformation into SCLC has been reported as an evolution of lung adenocarcinoma acquiring resistance to EGFR tyrosine kinase inhibitors (TKI) and is considered to be a rare resistance mechanism of EGFR-TKI therapy. We present a case of a 40 years old female presenting with cough and hemoptysis. Endobronchial lung biopsy was performed and it showed EGFR immunohistochemical expression. However, patient relapsed after successful treatment with erlotinib for 7 months, at which point second biopsy identified SCLC and chemotherapy with cisplatin and permetrexed was started. She is on regular follow up and is responding well to treatment. Our study highlights the importance of rebiopsy as transformation of the tumor can occur and correlates with the difference between SCLC and adenocarcinoma in sensitivity to therapies, so, repeated biopsies are beneficial for choosing appropriate treatments.
\end{abstract}

Keywords: Adenocarcinoma; Epidermal growth factor receptor (EGFR); Mutation; Small cell lung carcinoma; Transformation

\section{Introduction}

Lung cancer is the most frequently diagnosed cancer and leading cause among cancer mortality worldwide [1]. It arises from the cells of the respiratory epithelium and can be divided into two broad categories - small cell lung carcinoma (SCLC) and non-small cell lung carcinoma (NSCLC). SCLC is a highly malignant tumor derived from cells exhibiting neuroendocrine characteristics and accounts for $15 \%$ of lung cancer cases. NSCLC which accounts for the remaining $85 \%$ of cases is further divided into 3 major pathologic subtypes: adenocarcinoma (ADC), squamous cell carcinoma (SCC), and large cell carcinoma. ADC accounts for $38.5 \%$ of all lung cancer SCC for $20 \%$ and large cell carcinoma for $2.9 \%$ [2].

EGFR mutant lung adenocarcinoma eventually acquires drug resistance despite EGFR-Tyrosine kinase inhibitors therapy. Transformation to small-cell lung cancer (SCLC) is considered to be a rare resistance mechanism of EGFR-TKI therapy. Herein, we report a rare case of NSCLC transforming into SCLC with EGFR positivity. Our study also throws light on the need of second biopsy in these patients with transformation and drug resistance.

\section{Case Report}

A 40 years old female presented to chest outpatient department (OPD) with chief complaints of cough and hemoptysis. She was a non-smoker and had no obvious history suggestive of any malignancy. Chest X ray revealed few well circumscribed round opacities of size 1 to $3 \mathrm{~cm}$ in left lower zone with pleural effusion. Contrast enhanced computed tomography (CECT) thorax was performed, which showed heterogeneous moderately enhancing lesion in left lower lobe causing compression and narrowing of lower lobe and segmental bronchi along with pleural involvement (Figure 1). Endobronchial fine needle aspiration cytology (FNAC) was performed which revealed atypical cells arranged in groups, sheets, acini and rosettes in a hemorrhagic background. These cells were pleomorphic, with high nuclear cytoplasmic ratio, reticulogranular chromatin and inconspicuous nucleoli with scant to moderate amount of cytoplasm, suggestive of the diagnosis of adenocarcinoma lung (Figure 2). Further, endobronchial lung biopsy was received in our department for histopathological examination and 
immunohistochemistry (IHC). Diagnosis of adenocarcinoma was rendered. On immunohistochemistry (IHC), the tumor cells were positive for Cytokeratin, Napsin A, TTF-1 and EGFR (Figure 3). Patient was started on treatment with erlotinib (150 mg daily) as per the protocol which we follow in our institute. Within 5 months of treatment with erlotinib, she achieved excellent clinical response.

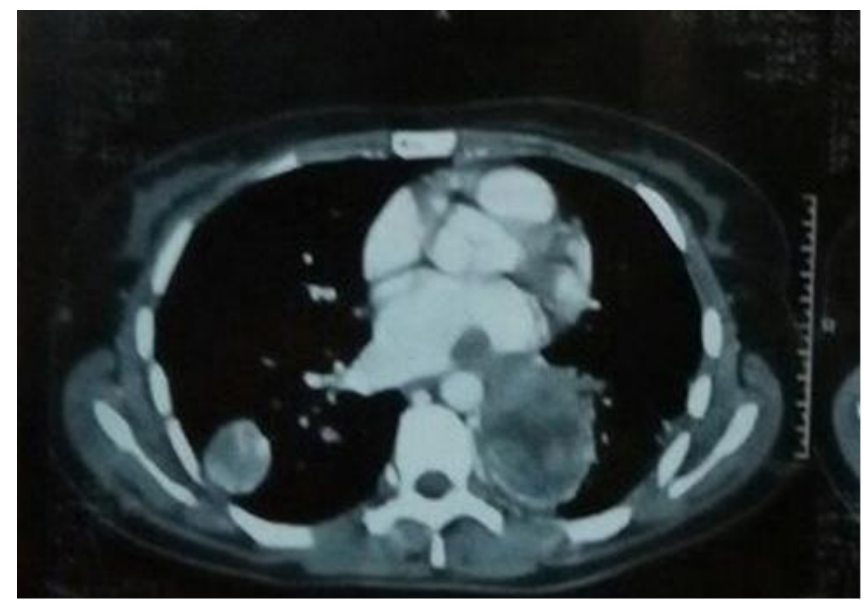

Figure 1: Contrast Enhanced Computed Tomography (Cect) Thorax Showing Heterogenous Moderately Enhancing Lesion with Bilateral Pulmonary Involvement and Collapse of Left Lower Lobe

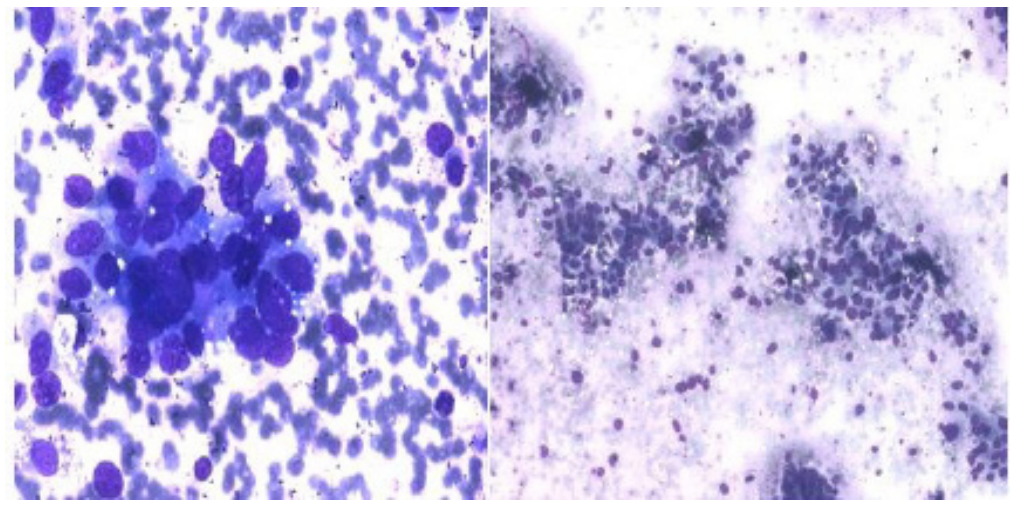

Figure 2: Fine Needle Aspiration Cytology (Fnac) Showing Highly Pleomorphic Tumor Cells Arranged In Acin and Rosettes

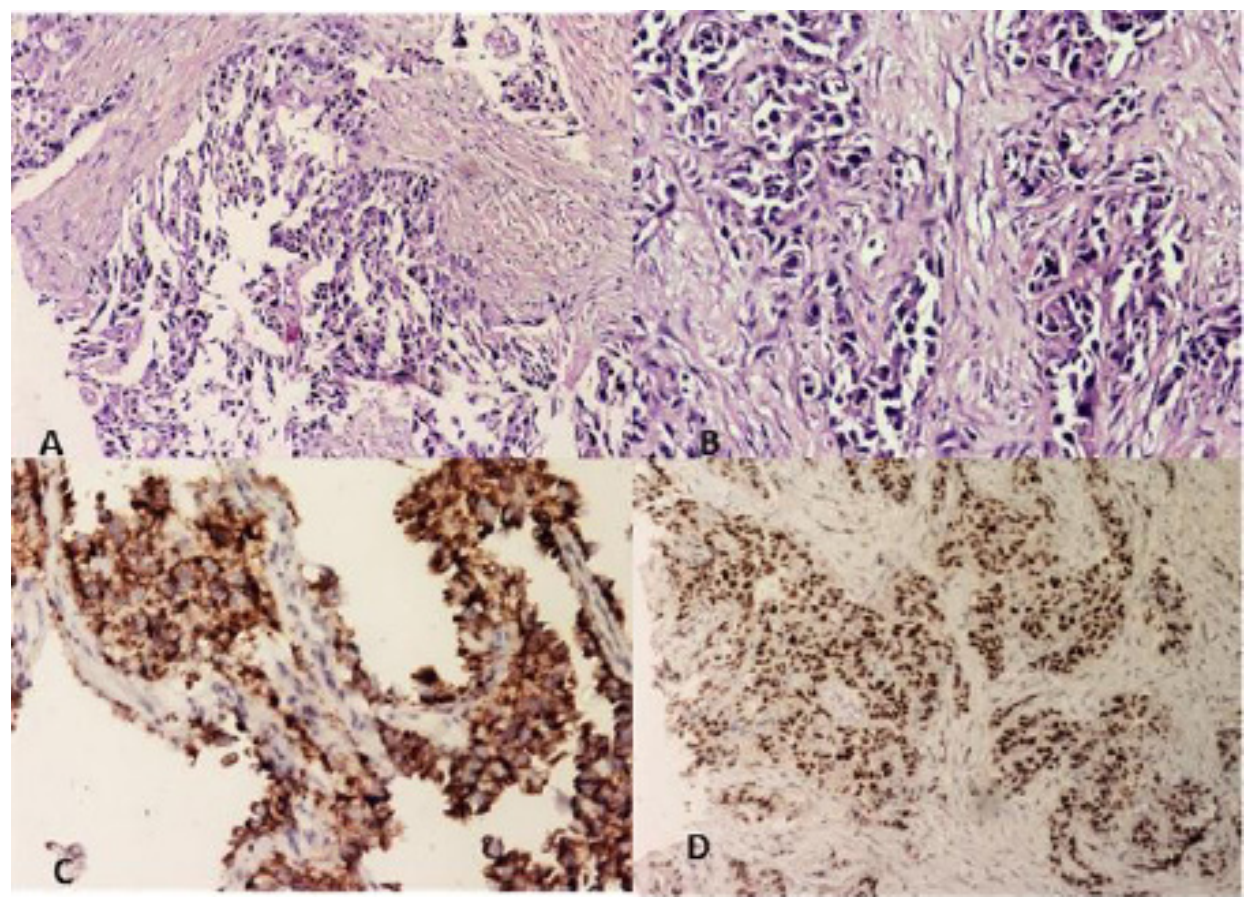

Figure 3: microscopy revealing adenocarcinoma, tumor cells arranged in glandular pattern $(\mathrm{a}, \mathrm{b})$, with positivity for napsin a (c), and ttf-1 (d) 
But later on, patient became unresponsive to treatment and her condition worsened. A repeat CECT thorax was advised which revealed a new nodule in lower lobe of left lung. A repeat endobronchial biopsy was performed and now showed features of small cell carcinoma (SCLC). On immunohistochemistry (IHC), tumor cells were CD 56 and synaptophysin positive. EGFR was strongly positive (Figure 4).

The patient is now being treated with cisplatin and permetrexed. She is on regular follow up and is responding well to treatment.

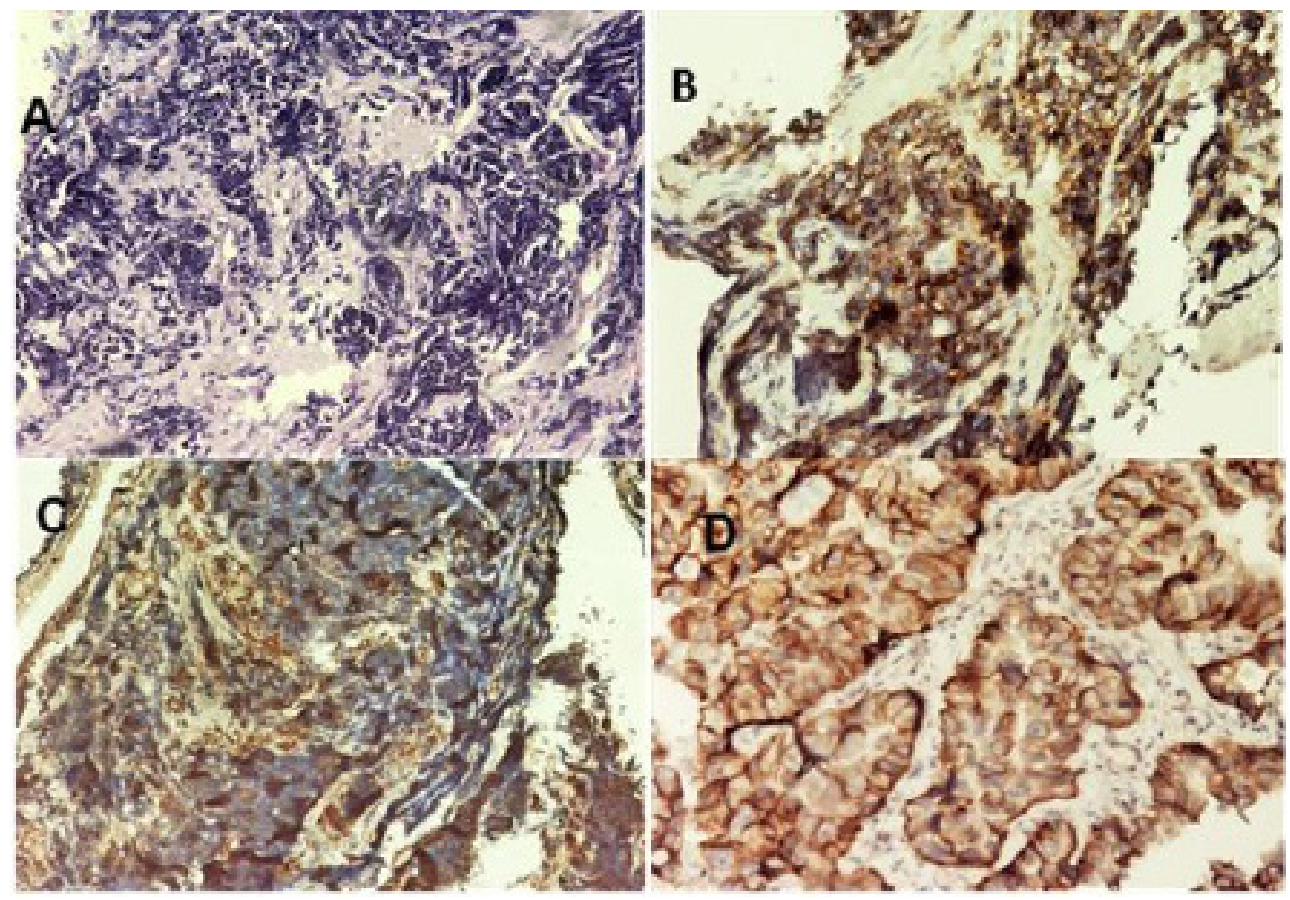

Figure 4: Photomicrograph Showing Histology Of Small Cell Carcinoma (A), Cd 56 (B), Synaptophysin (C), and Egfr (D) Positivity in Tumor Cells

\section{Discussion}

Although SCLC and NSCLC are two different entities owing to their distinct biology and genomic abnormalities, but the idea that is gaining support is that they might share common cell of origin. The fact which supports this is that a subset of NSCLC with mutated EGFR return as SCLC when resistance to EGFR- Tyrosine kinase inhibitors develops.

Epidermal growth factor receptor (EGFR) gene mutation is one of the most pervasive driver mutations in NSCLC, particularly adenocarcinoma [3]. Activating mutations in EGFR occur mostly in exons 18, 19 and 21 and are regarded as sensitizing mutations for EGFR tyrosine kinase inhibitors (TKIs) [4]. Epidermal growth factor receptor (EGFR)-tyrosine kinase inhibitors (TKIs) are highly promising drugs that are well tolerated and have good antitumor activity in patients with these patients [5]. Although, unfortunately their disease will ultimately develop drug resistance, which, despite extensive research, remains to be solved. EGFR mutations in SCLC mostly manifest as a "transformation" after EGFR-TKI therapy in EGFR-mutated adenocarcinoma, whereas primary SCLC with EGFR mutation is extremely rare [6].

The patient in our case was a middle-aged non-smoker female who was initially diagnosed with pulmonary adenocarcinoma. However, the disease transformed to SCLC and became resistant to erlotinib. Although the objective response to erlotinib therapy was modest, the dominant component of the tumor changed from adenocarcinoma to SCLC after EGFR-TKI therapy. Only a few cases have been reported in literature describing the primary EGFR-mutated adenocarcinoma transforming to SCLC [6,7]. Chronologically, the second SCLC is always perceived as "transformed SCLC". SCLC transformation from adenocarcinoma in patients with an EGFR mutation has been suggested as a mechanism of resistance to targeted therapy in the literature [8,9]. The incidence of SCLC in patients with adenocarcinoma is associated only with EGFR mutation status and not TKI use and the subsequent SCLCs were believed to either evolve from the adenocarcinoma or develop from a common precursor.

We suggest that responsiveness to EGFR-TKI varies between SCLC and adenocarcinoma. SCLC with an EGFR mutation is often resistant to EGFR-TKI, whereas most chemotherapies achieve good response in classical SCLC. SCLC component is more sensitive to cytotoxic chemotherapy than the adenocarcinoma component. The differences in effectiveness of therapeutic regimens between histological types strongly support the indication of repeated biopsies at the time of each treatment change. Second biopsy has been broadly recognized as necessary but rarely performed in daily practice due to imbalance between the potential risk of the diagnostic procedure and the therapeutic impact of the biopsy result [6]. Moreover, the most frequent mechanism of resistance to TKI is the occurrence of EGFR T790M mutation. Since this mutation can now be rapidly detected on a blood sample, re-biopsy is 
not the first thing to do in case of acquired resistance. Given the sensitivity of EGFR mutated SCLC to cytotoxic chemotherapies, re-biopsy for detecting tumor transformation can prove highly beneficial for patients. When SCLC occurs, the preexisting adenocarcinoma probably does not disappear. So SCLC must be treated with chemotherapy but TKI therapy should probably be maintained for the adenocarcinoma.

To summarize, 3 mechanisms are postulated to explain the switch between NSCLC and SCLC. Firstly, SCLC can result from the dedifferentiation of a previously well-defined cancer, which is similar to a mechanism known to occur in prostate cancer [10]. Secondly, both NSCLC and SCLC could arise from the same progenitor cell. The adenocarcinoma can differentiate and present as the first cancer. The activation of specific signalling pathways can lead to dormant stem cells undergoing SCLC transformation [11]. Thirdly, it is possible that the 2 components are present at initial diagnosis but the limited material available for analysis means that some synchronous cancers are mistaken as metachronous cancers, which may explain the occurrence of some combined cancers without a clear differential interval. Based on the available clinical information, these transformed SCLCs carry a relatively poor prognosis compared with classic SCLC. Whether these patients benefit from concurrent chemoradiotherapy requires further study. To understand the transformed subgroup better, further investigations are required to explore the molecular mechanisms of this transformation especially those involving the EGFR, RB1, and TP53 genes, and to identify the most appropriate treatment for these patients.

\section{Conclusion}

Our study extends the scope of SCLC developing in association with adenocarcinoma, either synchronously or metachronously. It suggests that these tumors are linked to EGFR mutations, regardless of TKI use as was concluded by Norkowski, et al. as well [12]. Thus, patients diagnosed with EGFR-mutant lung adenocarcinoma should be well supervised during the course of their disease with repeated biopsies because of the risk to develop combined SCLC or a recurrence with SCLC transformation. Furthermore, repeated biopsies are beneficial for choosing appropriate treatment according to the dominant histopathology. We suggest that cancers undergoing this transformation may represent a new subgroup of SCLC requiring further studies.

\section{References}

1. Jemal A, Siegal R, Xu J, Ward E (2010) Cancer statistics, 2010. CA Cancer J clin 60: 277-300.

2. Herbst RS, Heymach JV, Lippman SM (2008) Lung cancer. N Engl J Med 359: 1367-80.

3. Kris MG, Johnson BE, Kwiatkowski DJ, Iafrate AJ, Wistuba II, et al. (2011) Identification of driver mutations in tumor specimens from 1,000 patients with lung adenocarcinoma: the NCI's Lung Cancer Mutation Consortium (LCMC). J Clin Oncol 29: CRA7506.

4. De Pas T, Toffalorio F, Manzotti M, Fumagalli C, Spitaleri G, et al. (2011) Activity of epidermal growth factor receptor-tyrosine kinase inhibitors in patients with non-small cell lung cancer harboring rare epidermal growth factor receptor mutations. J Thorac Oncol 6: 1895-901.

5. Rossi A, Di Maio M (2015) LUX-Lung: determining the best EGFR inhibitor in NSCLC? Lancet Oncol 16: 118-9.

6. Sequist LV, Waltman BA, Dias-Santagata D, Digumarthy S, Turke AB, et al. (2011) Genotypic and histological evolution of lung cancers acquiring resistance to EGFR inhibitors. Sci Transl Med 3: 75ra26.

7. Morinaga R, Okamoto I, Furuta K, Kawano Y, Sekijima M, et al. (2007) Sequential occurrence of non-small cell and small cell lung cancer with the same EGFR mutation. Lung Cancer 58: 411-3.

8. Ma AT, Chan WK, Ma ES, Cheng T, Cheng PN (2012) Small cell lung cancer with an epidermal growth factor receptor mutation in primary gefitinibresistant adenocarcinoma of the lung. Acta Oncol 51: 557-9.

9. Popat S, Wotherspoon A, Nutting CM, Gonzalez D, Nicholson AG, et al. Transformation to "high grade" neuroendocrine carcinoma as an acquired drug resistance mechanism in EGFR-mutant lung adenocarcinoma. Lung Cancer 80: 1-4.

10. Walker GE, Antoniono RJ, Ross HJ, Paisley TE, Oh Y (2006) Neuroendocrine-like differentiation of non-small cell lung carcinoma cells: regulation by cAMP and the interaction of mac25/IGFBP-rP1 and 25.1. Oncogene 25: 1943-54.

11. D’Angelo SP, Pietanza MC (2010) The molecular pathogenesis of small cell lung cancer. Cancer Biol Ther 10: 1-10.

12. Norkowski E, Ghigna MR, Lacroix L, Le Chevalier T, Fadel É, et al. (2013) Small-cell carcinoma in the setting of pulmonary adenocarcinoma: new insights in the era of molecular pathology. J Thorac Oncol 8: 1265-71. 


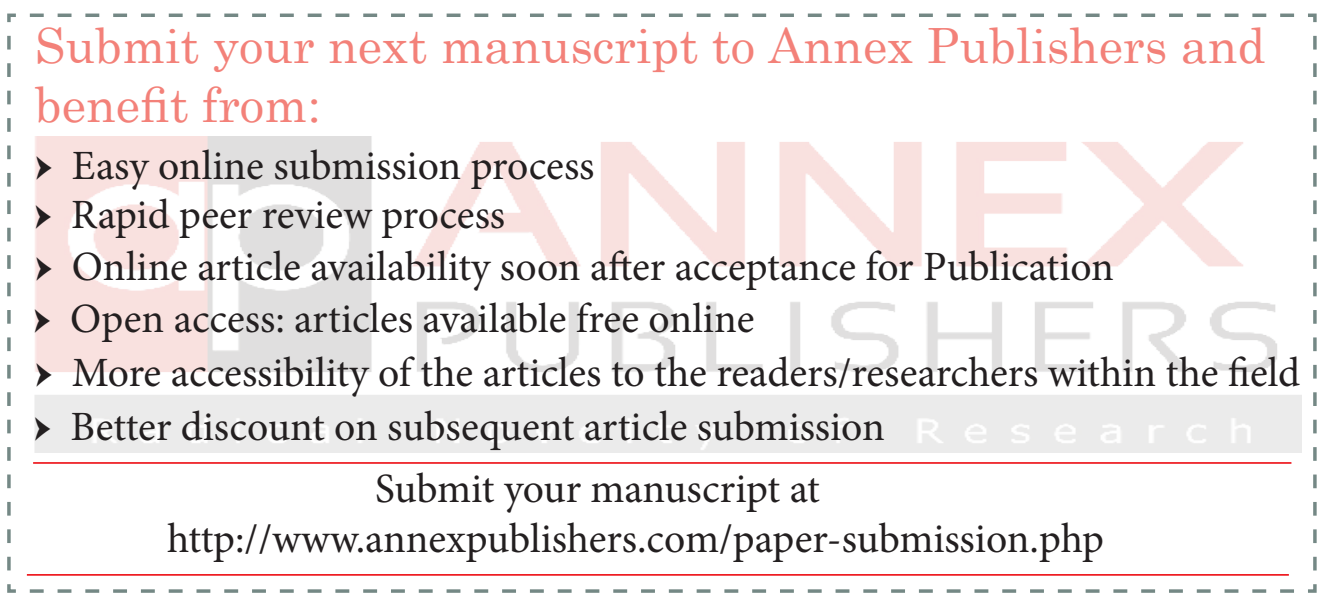

